\title{
Quantitative Support for Convergence of Intrinsic Energies from Applied Magnetic Fields and "Noise" Fluctuations of Newton's Gravitational Value Within The Human Brain
}

\author{
Michael A. Persinger*, Kevin S. Saroka \\ Laurentian University, Sudbury, Ontario P3E 2C6, Canada \\ *E-mail address: mpersinger@laurentian.ca
}

\begin{abstract}
When the energy within the mass of the human brain that is associated with the intrinsic range in fluctuations of the Gravitational Constant $(G)$ is set equal to the energy from a magnetic field (B) within the cerebral volume and solved for $\mathrm{B}$, a value in the order of 20 to $50 \mathrm{nT}$ is obtained. Quantitative Electroencephalographic (QEEG) and sLORETA (Low Resolution Electromagnetic Tomography) analyses of cerebral cortical activity during exposure to a range of applied rotating, frequency-modulated, transcerebral (between the two temporal lobes) magnetic fields between $<1 \mathrm{nT}$ and $7000 \mathrm{nT}$ while volunteers sat within a darkened, quiet chamber were completed. There was marked enhancement of power within the $4 \mathrm{~Hz}$ to $10 \mathrm{~Hz}$ band within the right caudal (cuneus) hemisphere while the $\sim 5$ to $20 \mathrm{nT}$ averaged strength magnetic fields were applied but no significant responses at lesser or greater intensities. These results suggest that a physical process coupled to the source of the fluctuation $\left(\sim 10^{-15} \mathrm{~m}^{3} \cdot \mathrm{kg}^{-1} \cdot \mathrm{s}^{-2}\right)$ in G may interact with right hemispheric activity within the range at which gravity waves have been estimated to interact with Schumann frequencies generated between the earth and ionosphere.
\end{abstract}

Keywords: Newton's G; "noise" fluctuations in G; gravitational-magnetic energy equivalents; cerebral magnetic field applications; QEEG; right hemisphere sensitivity; theta-alpha band activity

\section{INTRODUCTION}

The demonstration of the quantitative relationship between gravitational energy and electromagnetic energy has been considered important for the understanding of the mechanisms by which these two major universal qualities interact. Previously we [1] have shown that the intrinsic operational intensities of time-varying magnetic fields within the human cerebrum during complex processes such as cognition are within the picoTesla range and similar to those found within intragalactic space [2]. Such small magnitudes are similar to those found within the Schumann resonance [3], which displays a fundamental (first) harmonic of $\sim 7.8 \mathrm{~Hz}$ with amplitudes of about 2 picoTesla, that is generated within the earth and ionosphere cavity [4].

The gravitational energy within the human cerebrum can be estimated by the relation:

$$
\mathrm{J}=\mathrm{G} \cdot \mathrm{kg}^{2} \cdot \mathrm{m}^{-1}
$$


where $\mathrm{G}$ is the Newtonian Gravitational Constant $\left(6.67 \cdot 10^{-11} \mathrm{~m}^{3} \cdot \mathrm{kg}^{-1} \cdot \mathrm{s}^{-2)}, \mathrm{kg}\right.$ is mass of the cerebrum and $\mathrm{m}$ is its average length. If the mass of $1.35 \mathrm{~kg}$ and an average length of $1.1 \cdot 10^{-1}$ $\mathrm{m}$ (based upon the cube root of its volume of about $1330 \mathrm{cc}$ ) are assumed, the energy within the human cerebrum is $\sim 1.1 \cdot 10^{-9} \mathrm{~J}$.

If each action potential $\left(\Delta \mathrm{V}=1.2 \cdot 10^{-1} \mathrm{~V}\right)$ exerts upon each unit charge $\left(1.6 \cdot 10^{-19} \mathrm{~A} \cdot \mathrm{s}\right)$ an increment of energy of $1.9 \cdot 10^{-20} \mathrm{~J}$ [5], then the energy associated with gravitation within the human cerebrum would have the capacity to support the activity of $10^{11}$ neurons or $10^{10}$ neurons each discharging on average around $10 \mathrm{~Hz}$. There are approximately 20 billion neurons in the cerebral cortices, which suggest that conditions associated with G might set the boundary for some component of neuronal potential within the human cerebral cortices.

However $G$ is not constant. Previous and more recent measurements [6] have shown that there are changes or fluctuations in $\mathrm{G}(\Delta \mathrm{G})$ in the order of $5 \cdot 10^{-14} \mathrm{~m}^{3} \cdot \mathrm{kg}^{-1} \cdot \mathrm{s}^{-2}$, which results in a value of $8.3 \cdot 10^{-13} \mathrm{~J}$. The presence of this order of magnitude of perturbation in $\mathrm{G}$ is relevant. This quantity would be associated with about $10^{7}$ neurons each discharging with $10^{-20} \mathrm{~J}$ per action potential approximately once per second. This number approaches the critical mass of neurons required for "awareness" and "ideations".

Considering the volume of the human cerebrum is $\sim 1.3 \cdot 10^{-3} \mathrm{~m}^{3}$ the energy from gravitational fluctuations will be $\sim 0.6 \cdot 10^{-9} \mathrm{~J} \cdot \mathrm{m}^{-3}$, the approximate average energy density of the universe [7]. In many systems a unit with the same potential energy as the field in which it is immersed has the capacity for some form of resonance, particular near boundary or threshold intensity values.

If one assumes a unit $\mathrm{Hz}$ for this gravitational energy, the radiant flux density from the cross sectional area of the human cerebral cortices $\left(1.2 \cdot 10^{-2} \mathrm{~m}^{2}\right)$, assuming $8.2 \cdot 10^{-13} \mathrm{~J} \cdot \mathrm{s}^{-1}(\mathrm{~W})$ would be $\sim 7 \cdot 10^{-11} \mathrm{~W} \cdot \mathrm{m}^{-2}$. If the implicit variation was less than $1 \mathrm{~Hz}$, for example $10^{-1} \mathrm{~Hz}$ or in the range of micropulsations, the radiant flux density equivalence would be $0.7 \cdot 10^{-11} \mathrm{~W} \cdot \mathrm{m}^{-}$ ${ }^{2}$. We [8] have also measured a direct coupling between background photon emissions and geomagnetic activity. For every $1 \mathrm{nT}$ decrease in geomagnetic activity there is a $10^{-12} \mathrm{~W} \cdot \mathrm{m}^{-2}$ increase in photon emission. When one assumes a frequency for an upper limit $(20 \mathrm{mHz})$ for the earth's free oscillations the gravitational energy equivalent within the human cerebrum would be $1.4 \cdot 10^{-12} \mathrm{~W} \cdot \mathrm{m}^{-2}$. Such flux densities have been measured from sections of hippocampus tissue.

One lower limit for the boundary for the net shift in $\Delta \mathrm{G}$ as measured by Vladimirskii [9] for days with $A_{p}$ values of $<8$ or $>30$ was $5 \cdot 10^{-15} \mathrm{~m}^{3} \cdot \mathrm{kg}^{-1} \cdot \mathrm{s}^{-2}$. That would be associated with a gravitational energy within the cerebral mass of $8.3 \cdot 10^{-14} \mathrm{~J}$. If the topological nature of the human cerebral cortices is considered, where two-thirds is buried below the observable surface, the true surface area is $1.8 \cdot 10^{-1} \mathrm{~m}^{2}$. The "energy density" would be $4.6 \cdot 10^{-13} \mathrm{~J} \cdot \mathrm{m}^{-2}$. If the interface between gravitational waves and electromagnetic waves is the second Schumann harmonic of $14 \mathrm{~Hz}$ [10] which is also the electroencephalographic band associated with the transition between relaxation and cognitive activation, the flux density would be $0.6 \cdot 10^{-11}$ $\mathrm{W} \cdot \mathrm{m}^{-2}$.

This precise order of magnitude and coefficient have been measured by photomultiplier units from the human cerebrum when people engage in imagination but not more mundane cognitive activities [9]. This convergence suggests that the threshold for $\Delta \mathrm{G}$ has the capacity under optimal conditions to interact with the nature of the photon emissions associated with cerebral function in general and the process of "thinking" in particular.

To test this concept, we designed an experiment where different intensities of bioeffective, time-varying magnetic fields that: 1 ) have been shown to be equivalent to $4 \mathrm{mg}$ per 
$\mathrm{kg}$ of morphine for analgesia in rodents and 2) delay the growth of cancer cells, were exposed trancerebrally (across the temporal lobes). Specific changes in current density within specific frequency bands were measured as a function of the intensities. We predicted the specific strengths of applied magnetic fields that would interact with the gravitational energy potentials. Here we present evidence that this interaction is robust and is demonstrable in the laboratory.

\section{CAlCUlations}

Helmholtz' principle that energy is neither created nor destroyed but only modified in form permits different derivations of energy to be set as equivalents. Consequently the energy associated the magnetic field within a volume and that associated with the coupled mass is equated by:

$$
\left(B^{2}\right) \cdot(2 \mu)^{-1} \cdot \mathrm{m}^{3}=\mathrm{G} \cdot \mathrm{kg}^{2} \cdot \mathrm{L}^{-1}
$$

where, $B=$ the magnetic field strength, $\mu=$ magnetic permeability of a vacuum $\left(4 \pi \cdot 10^{-7} \mathrm{~N} \cdot \mathrm{A}^{-}\right.$ $\left.{ }^{2}\right), \mathrm{G}$ is the Newtonian Gravitational Constant $\left(6.67 \cdot 10^{-11} \mathrm{~m}^{3} \cdot \mathrm{kg}^{-1} \cdot \mathrm{s}^{-2)}, \mathrm{kg}\right.$ is the mass of the cerebrum and $\mathrm{L}$ is its average length. If one assume the average volume of $1.3 \cdot 10^{-3} \mathrm{~m}^{3}$, mass of $1.5 \mathrm{~kg}$, and $\mathrm{L}=1.1 \cdot 10^{-1} \mathrm{~m}(11 \mathrm{~cm})$ and solves for $\mathrm{B}$, the resulting value is $1.5 \cdot 10^{-6} \mathrm{~T}$.

In other words, the equivalent strength of energy as a magnetic field within the volume of the human cerebrum that reflects its mass is $15.5 \mathrm{mG}$. This is well within the range of intensities [10] that when applied through the human brain as appropriately (physiologically) patterned magnetic fields produce significant alterations in subjective experiences as well as shifts in current densities involving different frequency bands as measured by sLORETA (Low Resolution Electromagnetic Tomography).

On the other hand the equivalent magnetic field strength associated with the average variation of $5 \cdot 10^{-14} \mathrm{~m}^{3} \cdot \mathrm{kg}^{-1} \mathrm{~s}^{-2}$ would be $\sim 5 \cdot 10^{-8} \mathrm{~T}$ or $50 \mathrm{nT}$. If the boundary for threshold variations discerned by Vladimirskii [9] is applied the effective variation would be $\sim 17 \mathrm{nT}$. We reasoned that if the effect was as fundamental as inferred, then a narrow range of intensities induce significant shifts in power within the cerebrum within the range of the Schumann resonance. The maximum response for either the strongest or weakest fields could be instructive for revealing potential connections between gravitational forces and cerebral functioning.

\section{METHODS AND PROCEDURES}

One young male and female volunteer (ages 25 to 28 years) volunteered to participate. We reasoned that if the predictions were accurate the effect should be similar and conspicuous with only two subjects when exposed to a repeated measures experimental procedure where each person was his or her own control. The circumferences of the man's and woman's skull were $66 \mathrm{~cm}$ and $57 \mathrm{~cm}$, respectively. Each subject was exposed to our protocol that has been approved by the university's Research Ethics Board. In summary each person sat while blindfolded in a comfortable chair in a darkened acoustic chamber that was also a Faraday room. The static geomagnetic field was reduced by about one-half (to $\sim 25,000 \mathrm{nT}$ ) within this setting. 
Each subject had been fitted with a 19-channel EEG cap (Electro-Cap International) that was connected to a Mitsar-201 amplifier. The amplifier was connected to an Apple Macbook running Microsoft Windows 7 via USB cable. The placement of the 19 sensors (Fp1, Fp2, F7, F3, Fz, F4, F8, T3, C3, Cz, C4, T4, T6, P3, Pz, P4, T6, O1 and O2) was in accordance with the 10-20 International Standard of Electrode Placement. All sensor impedances were kept below 5 kiloOhms. All data was visualized, recorded, filtered and artifact-corrected with WinEEG v 2.89.52 software for Microsoft Windows. The activity was sampled at $250 \mathrm{~Hz}$.

After an initial eyes-closed baseline was collected each of the two participants received different intensities of a frequency-modulated electromagnetic field delivered by the SAM360 device, the details of which have been previously described in Persinger and Saroka [11]. The duration of exposure for each intensity was two minutes. Each box of the SAM-360 was placed over the temporo-parietal region on the left and right hemispheres. The canisters, each containing four solenoids (reed switches), were attached by Velcro to each side of the person's head above the ears. The pairs of solenoids generated the magnetic field through the intervening cerebral space with minimal attenuation [11 ].

The intensities were controlled with Complex 2, a custom-designed application operating in DOS that generates complex time-varying electromagnetic signals. The QEEG equipment continuously monitored each participant's brain activity during the entire experiment. During the approximately 40 minutes of the experiment, each of between 6 and 7 different intensities between $<1 \mathrm{nT}$ and $7000 \mathrm{nT}$ of the physiologically patterned magnetic field were generated continuously for 6 min each across the hemispheres. The interval of no field between presentations was about $30 \mathrm{~s}$. The duration of individual exposures was set equal to 6 min so that we could include most of the known intrinsic "gravitational" modes between the solid earth and atmosphere.

The specific configuration, a frequency-phase modulated pattern, was selected for the field configuration. It was the initial component for a protocol for establishing excess correlations between photon-generating chemical reactions. The visualization of the pattern has been published many times elsewhere [12]. It has been employed to alter consciousness and is also known to affect the nociceptive thresholds of rodents [12] and planarian [13]. The sequence was composed of 849 numbers between 0 and 255 .

Each of the 849 numbers between 0 and 255 was transformed by a custom-constructed digital-to-analogue computer to a value between -5 and $+5 \mathrm{~V}$. The duration each number (voltage) was activated for $3 \mathrm{~ms}$. This was essential because other research involving cellular responses to this field pattern indicated a maximum effect with $3 \mathrm{~ms}$ point durations while those values less $(1 \mathrm{~ms}, 2 \mathrm{~ms})$ or greater $(4 \mathrm{~ms}, 5 \mathrm{~ms}$, or $7 \mathrm{~ms})$ were not effective.

The entire quantitative electroencephalographic record for Participant 1 (male) was segmented into 10-second increments. These segments were then entered into sLORETA software for source localization. A preliminary analysis involving the "Statistics" function within SLORETA [14] was performed by comparing the brain activity recorded during one of the intensity measures against an initial eyes-closed baseline.

To replicate the effect observed with Participant 1, the entire EEG record for Participant 2 (female) was parsed into 10-second segments and entered into sLORETA to obtain the current source densities for the right cuneus involved with same region found for Participant 1. These data were analyzed separately within SPSS. The one-way analyses of variance were performed with current source densities of specific classical bands as a function of electromagnetic field intensity. 
Before and after the experiment the two containers were separated by the distance of the diameter of the skulls of the two participants. The magnetic field values for each of the different intensities to which the person had been exposed were measured through a power meter interfaced with computer software within a laptop computer. The mean intensity for each increment was obtained for the midposition between the two canisters and along the caudal boundaries that would have been occupied by the participant's cerebrum. The field strengths were measured with a power meter and its digital output as well as with a MEDA magnetometer for independent verification.

We appreciated the attenuated sensitivity for the fast transients would be attenuated within the magnetometer. It was employed primarily for verification of the field strengths below the background ubiquitous $60 \mathrm{~Hz}$ which was 18 to $20 \mathrm{nT}(0.18$ to $0.20 \mathrm{mG})$. Within 2 $\mathrm{cm}$ (approximately the area of the cerebral cortices), the peak strengths ranged from $20 \mu \mathrm{T}$ to $8 \mathrm{nT}$ for the different settings. Within the middle of the cerebral space, equidistant between the two field sources, the field strengths ranged from $2 \mu \mathrm{T}$ to $<1 \mathrm{nT}$.

\section{RESULTS}

The region with the most significant increase in power during stimulation by the optimal strength of the applied magnetic field occurred within the region of the right caudal hemisphere for both subjects. More specific coordinates indicated the volume of cerebrum was traditionally labeled the cuneus within the right hemisphere.

Even though each subject was exposed to a variety of intensities between $<1 \mathrm{nT}$ and 7 microTesla, the most significant increase in power density within the electroencephalographic activity within the right cuneus occurred for the narrow band between $\sim 5$ to $20 \mathrm{nT}$ that was within the range predicted by the equation when the background fluctuation in $G$ was considered. The results are shown in Figure 1. The frequency range that was most affected involved the upper theta to alpha band. The difference in peak frequency between the two subjects was consistent with their estimated cerebral circumferences which, assuming a classic bulk velocity of integrated cortical electromagnetic fields to be about $4.5 \mathrm{~m} \cdot \mathrm{s}^{-1}$ to obtain the 20 to $25 \mathrm{~ms}$ recursive rate associated with ("40" $\mathrm{Hz}$ ) consciousness would be 7.9 $\mathrm{Hz}$ and $6.8 \mathrm{~Hz}$, respectively.

The analysis for Participant 1 demonstrated a significant spatial effect $(p<.05)$ whereby there was more power for alpha $(7-10 \mathrm{~Hz})$ activation within the right cuneus during the lowest intensity electromagnetic field application. To compare activation within this specific region of the brain across all of the intensities of the same electromagnetic field pattern, the saved current source densities $\left(\mu \mathrm{A} \cdot \mathrm{mm}^{-2}\right)$ were imported into SPSS.

One way-analyses of variance, completed for current source densities within the right cuneus across all classical frequency bands: delta $(1.5-4 \mathrm{~Hz})$, theta $(4-7 \mathrm{~Hz})$, low-alpha (7-10 $\mathrm{Hz})$, high-alpha $(10-13 \mathrm{~Hz})$, beta-1 $(13-20 \mathrm{~Hz})$ beta-2 $(20-25 \mathrm{~Hz})$ beta-3 $(25-30 \mathrm{~Hz})$ and gamma $(>30 \mathrm{~Hz}))$, demonstrated statistically significant enhancement of power only within the theta-alpha band interface. For Participant 2, the right cuneus was also the region that displayed the enhancement but the peak occurred within the theta $(4-7 \mathrm{~Hz})$ band.

Subsequent to the analyses of the data that showed that the region of the right precuneus (which displayed the most conspicuous shift in power in response to the application of the magnetic field) would have been within the area where the strength of the applied, patterned magnetic field was within the $<1 \mathrm{nT}$ to $5 \mathrm{nT}$ range. However these values were below that of 
the background levels of the power meter and were discerned by the magnetometer which would have not been as sensitive to the higher frequency components.
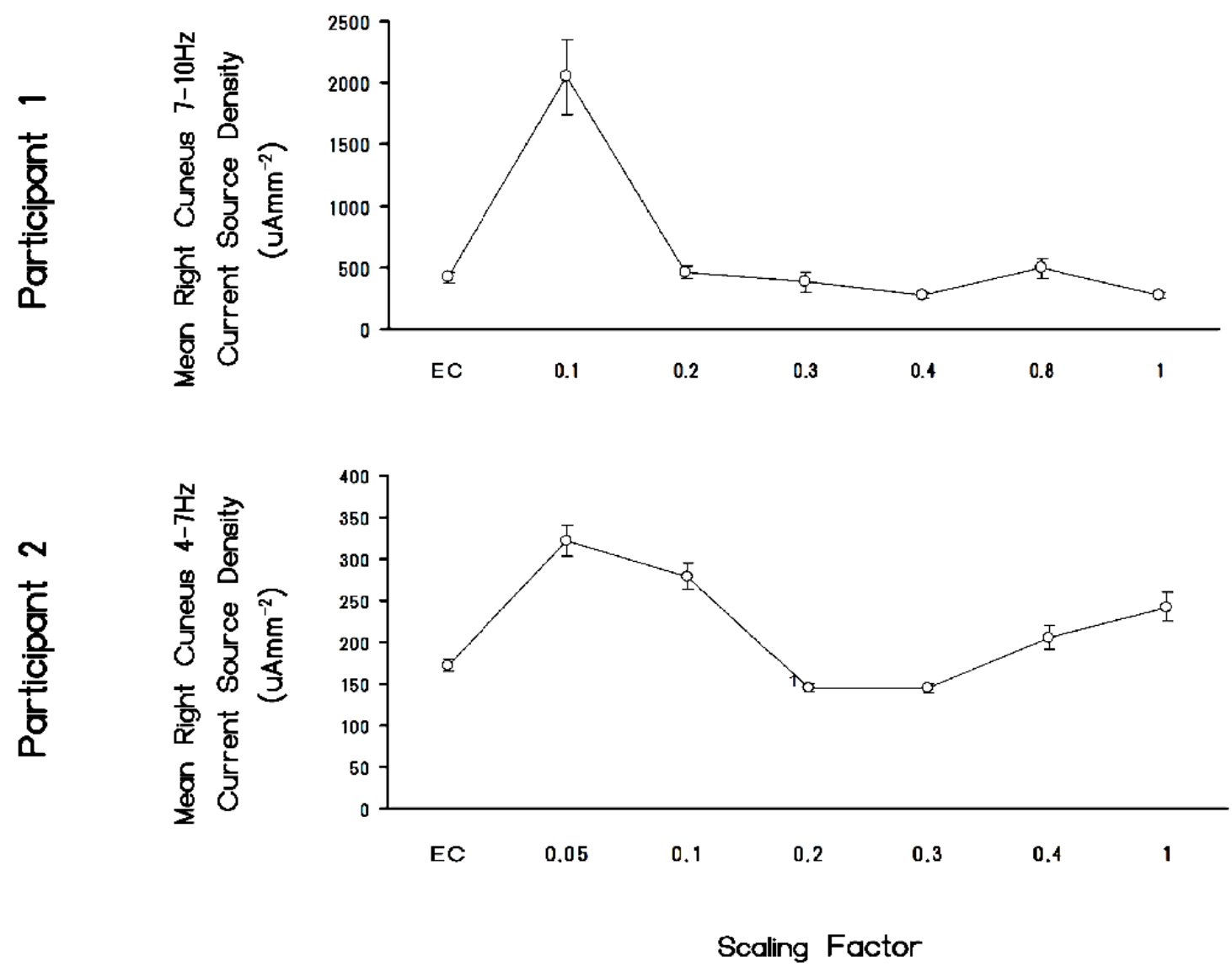

Figure 1. Current densities within the right cuneus region of the brains of two participants who were exposed to brief frequency (phase)-modulated magnetic fields across the temporal lobes. EC refers to eyes closed (no field, $<1 \mathrm{nT}$ ). The numbers indicate the multiplication factor from the software employed to generate the field. The equivalent averaged values within most of the cerebral volume: in nanoTesla $(\mathrm{nT})$ were $0.05(5 \mathrm{nT}), 0.1(20 \mathrm{nT}), 0.2(50 \mathrm{nT}), 0.3(170 \mathrm{nT}), 0.4(230 \mathrm{nT}), 0.8(1.4 \mu \mathrm{T})$ and $1.0(1.8 \mu \mathrm{T})$.

\section{DISCUSSION}

The concept that energy is neither created nor destroyed but changes form becomes applicable biologically when one form of energy is set equal to another and the characteristics of the alternative energy is pursued. In this instance the energy associated with G was set equal to the energy associated with a magnetic field. This resulted in two solutions for the equivalent magnitude. The first, associated with $G$ proper, was an equivalent quantity reflected as 1.5 microTesla. The second was associated with the threshold for variation in $G$ and was associated with an intensity of $\sim 5$ to $20 \mathrm{nT}$.

The results of our experimental data indicate that the interface occurs with some process associated with the threshold fluctuations in $\mathrm{G}$, which is in the order of $5 \cdot 10^{-14}$ to $5 \cdot 10^{-15} \mathrm{~m}^{3}$ $\cdot \mathrm{kg}^{-1} \cdot \mathrm{s}^{-2}$, that within the mass and volume of the human cerebrum is $\sim 30$ to $17 \mathrm{nT}$. Within this interface the current source densities within a specific volume of the cerebrum, the right 
cuneus, increased by a factor of 2 to 4 compared to the no experimental field (eyes closed) condition or to the other intensities of the same pattern of magnetic field that were all generated across the temporal lobes. Subsequent measurements within the approximate space where the cerebrums would be located indicated that the average field strength would have been within the predicted range.

The maximum response for intensities that were congruent with magnetic energy equivalents of $\Delta \mathrm{G}$, the intrinsic variation in $\mathrm{G}$ rather than the Newtonian constant might suggest that the variance in spatial structure responsible for the oscillations or the "noise" in the measurement of $G$ that have frustrated physicists for several decades [6], may be directly interactive with brain function. The approximate energy equivalent to about $17 \mathrm{nT}$ is within error measurement of the $\pm 10 \mathrm{nT}$ variations in the magnetic intensity of the solar wind that was found by Vladimirskii [8] to be associated with $\Delta \mathrm{G}$. They found, as did Persinger and StPierre [6] that as the magnetic fluctuations increased the amplitude of $\Delta \mathrm{G}$ decreased.

The large effect between 5 and $20 \mathrm{nT}$ was below the background $60 \mathrm{~Hz}$ power frequency fields that pervade the environment. These results suggest that the pattern of the field, in this case the frequency-modulated signaling nature of the configuration when applied across the two hemispheres at the level of the temporal lobes is more involved with the gravitational-electromagnetic interaction than simply amplitude.

The effectiveness of analogous "subthreshold" patterns in nature, perhaps hidden as stochastic sources of resonance, could require a re-evaluation of the importance of calculated field strengths that are below measurement background. An analogous consideration has been discussed by Cifra and his colleagues [15]. They argued that the classic explanation that microTesla and nanoTesla magnetic field strengths cannot be effective because they are below the average energy of thermal agitation according to the (Boltzmann) "kT constraint", was not applicable to living systems because they are not in thermal equilibrium.

The contribution from $\mathrm{G}$ per se to the cerebral effect, if it were present, was much weaker. As can be seen in Figure 1, the current densities of the enhanced frequency within both subjects were significantly higher (between 2 and $18 \mathrm{mG}$ ) than the no field or base line condition. The average equivalent intensity $(15 \mathrm{mG})$ would have been within the range of that predicted by the energy relationship. However it was much weaker than the effect predicted for the energy relationship with $\Delta \mathrm{G}$.

We had not predicted where within the cerebral volume the maximum change in power would occur. The results of the study indicate that the caudal portion of the human brain within the right hemisphere and particularly along the medial surface was most affected by the application of the experimental magnetic fields across the entire cerebrum. We have found in our large data base of QEEG and LORETA configurations for normal people that Schumann resonances are more discernable over the caudal portions of the human cerebrum relative to middle and frontal regions. The specific area, the cuneus, is located caudal to the parieto-occipital sulcus and dorsal to the calcarine fissure. With the exception of the approximately $10 \%$ greater relative proportion of white matter and blood flow within the right hemisphere, in general, there is no conspicuous structural feature of this region.

The right cuneus is associated with complex visual processing that couples attention with reward and memory. Traditionally the right cuneus should respond maximally to visual stimuli directed to the upper left retinal field (lower left visual field), although there is also evidence that the right upper retinal field (lower right visual field) projects substantially to the lower half of the right cuneus. Diminished density of neuronal soma (grey matter) within the right cuneus has been found in first onset psychosis [16] and greater regional homogeneity for MRI (Magnetic Resonance Imaging) signals have been measured for patients diagnosed with 
panic disorders. Increased co-activation between the ventrolateral nuclei of the thalamus and the right cuneus occurs when patients diagnosed with Post-Traumatic Stress Disorder (PTSD) begin to display dissociative responses [17].

Individuals, including older children, who have been diagnosed with Type I diabetes and have been exposed to repeated episodes of hypoglycemia exhibit less grey matter within the right cuneus [18]. However dynamic changes in this region are not always associated with pathology. Number multiplications and the facility with arithmetic manipulations are associated with activation of this region but also include the left and right parietal region, left fusiform area, and lingual gyri [19]. These behavioral correlates are presented as indicators for future studies.

The more profound significance of the right cuneus in the gravitational-magnetic equivalence, assuming the effect is generalizable, remains to be discovered. The amplification of power within this region that was measured in the present study may not be a direct effect. The whole cerebrum was exposed to our magnetic field configurations. Consequently the cuneus effect may reflect how the cerebrum responds to these energy sources.

The peak increases in current distribution with the right hemisphere suggests that components of any information contained within the interface between the threshold for $\Delta \mathrm{G}$ and the $\sim 5 \mathrm{nT}$ magnetic fluctuations may not be reflected in consciousness or awareness. These cognitive phenomena are primarily left hemispheric processes. However, because dreaming (Rapid Eye Movement) reflects more right hemispheric dominance, the probability would increase that any such information from $\Delta \mathrm{G}$ and $\sim 5 \mathrm{nT}$ patterned magnetic fluctuations could be manifested within that state which could subsequently affect behaviors the following day or days.

Scott and Persinger[20] found that subtle discernment of stimuli in the environment increased during days when geomagnetic activity averaged around $5 \mathrm{nT}$. They calculated that the amount of magnetic energy within the human cerebral volume would be in the order of $10^{-}$ ${ }^{14} \mathrm{~J}$. Assuming the Lindauer Limit values for the loss or gain information from entropy or from the random vacuum fluctuations to be about $2.95 \cdot 10^{-21} \mathrm{~J}$ per bit or unit convergence per "computation", this energy could transfer potentially about $3 \cdot 10^{6}$ bits. These authors argued that the optimal effect at $5 \mathrm{nT}$ suggested the presence of a natural band of interaction that required a discrete amplitude of energies.

The physical chemical mechanism within brain space by which the gravitationalmagnetic energy equivalence might be mediated could be coupled to the fundamental properties of water, particularly one of its mobile constituents: the proton. We have shown experimentally that the most likely physical mechanisms involved with effects of this magnitude of magnetic fields and temporal patterns involve the movements of protons from hydronium ions that compose the water of cerebral tissue [21-24]. Those basic physical constants that determine the characteristics of water have been shown previously. The ratio of the proton's magnetic moment $\left(1.41 \cdot 10^{-26} \mathrm{~A} \cdot \mathrm{m}^{2}\right)$ to its unit charge $\left(1.6 \cdot 10^{-19} \mathrm{~A} \cdot \mathrm{s}\right)$ results in a diffusivity term: $0.88 \cdot 10^{-7} \mathrm{~m}^{2} \cdot \mathrm{s}^{-1}$.

When multiplied by the viscosity at biological temperatures, $6.3 \cdot 10^{-4} \mathrm{~kg} \cdot \mathrm{m}^{1} \cdot \mathrm{s}^{-1}$, the force is $5.54 \cdot 10^{-11}$ Newtons. When applied across the distance of two O-H bonds $\left(1.92 \cdot 10^{-10}\right.$ $\mathrm{m})$, the energy is $1.1 \cdot 10^{-20} \mathrm{~J}$. This is equivalent to the range of the typical voltage of the resting membrane potential $(\sim 70 \mathrm{mV})$ or the peak-to-peak differences in action potential for burst-firing neurons [5].

The potentially direct role of the hydronium ion becomes evident when the mass-charge density is related to the $15.5 \mathrm{mG}$ energy equivalence between magnetic and gravitational sources. The mass of the $19 \mathrm{D} \mathrm{H}_{3} \mathrm{O}^{+}$is $3.15 \cdot 10^{-26} \mathrm{~kg}$ and when divided by the unit charge of 
$1.6 \cdot 10^{-19} \mathrm{~A} \cdot \mathrm{s}$, results in $1.97 \cdot 10^{-7} \mathrm{~kg} \cdot \mathrm{A}^{-1} \mathrm{~s}^{-1}$. When the $1.5 \cdot 10^{-6} \mathrm{~T}\left(\mathrm{~kg} \cdot \mathrm{A}^{-1} \cdot \mathrm{s}^{-2}\right)$ of energy equivalence is divided by the mass-charge ratio, the frequency approaches $7.8 \mathrm{~Hz}$. This the fundamental frequency of the Schumann Resonance as well as the first peak power mode in the cerebral cortical quantitative electroencephalographic (QEEG) profile.

However in the present experiments we found the most potent effects from the optimally applied experimental magnetic field matched the energies associated with the fluctuations of G. For $20 \mathrm{nT}$, the resulting resonance would be $0.1 \mathrm{~Hz}$. For $2 \mathrm{nT}$, the frequency would about $25 \mathrm{mHz}$. The latter periodicities are within the upper band of the free oscillations of the earth. The product of the mass of the human cerebrum and the amplitude of the fundamental spheroidal modes is about $0.5 \cdot 10^{-11} \mathrm{~m} \cdot \mathrm{s}^{-2}$. When the upper limit of $25 \mathrm{mHz}$ is multiplied the result is $2.3 \cdot 10^{-13} \mathrm{~kg} \cdot \mathrm{m} \cdot \mathrm{s}^{-3}$. Division by the averaged length of the cerebrum $(0.1 \mathrm{~m})$ produces a flux density of $2.3 \cdot 10^{-12} \mathrm{~W} \cdot \mathrm{m}^{-2}$. This is within the range of radiant power density of photon emissions from the cerebrum during some forms of cognition.

\section{CONCLUSION}

From the perspective of an integrated chemistry, physics and astronomy, there should an interface between gravitational and electromagnetic energies with specific quantitative properties. The equivalent energy as defined by traditional equations between the magnitude of the variation in $\mathrm{G}$ and electromagnetic forms indicates that the interface within the human cerebral volume is in the order of $20 \mathrm{nT}$. The most optimal change in the three-dimensional current densities within the human cerebrum was measured when physiologically-patterned electromagnetic fields within this range of intensity were applied across the cerebrum at the level of the temporal lobes. The responsiveness of the cerebrum to intensities as low as $5 \mathrm{nT}$ suggests that the observations of co-variation between $\mathrm{G}$ and solar wind intensities within the $5 \mathrm{nT}$ range could directly interface within cerebral space. The prominence of the right hemispheric response indicates the information may not always be reflected in predominately left hemispheric awareness but could be more easily acquired during states that emphasize right hemispheric activity such as dreaming.

\section{References}

[1] M. A. Persinger, B. T. Dotta, K. S. Saroka, M. A. Scott, Journal of Consciousness Exploration \& Research 4 (2013) 1-24.

[2] S. Redfield, J. Linsky, Astrophysics Journal 583 (2008) 283-314.

[3] M. A. Persinger, International Letters of Chemistry, Physics and Astronomy 11 (2014) 24-32.

[4] H. L. Koenig, A. P. Krueger, S. Lang, W. Sonning, Biologic Effects of Environmental Electromagnetism. Springer-Verlag, New York, 1981.

[5] M. A. Persinger, Current Medicinal Chemistry 17 (2010) 3094-3098.

[6] M. A. Persinger, L. S. St-Pierre, International Journal of Geosciences 5 (2014) 450-452.

[7] M. A. Persinger, International Journal of Astronomy and Astrophysics 4 (2014) 178-180. 
[8] D. A. E. Vares, M. A. Persinger, Journal of Non-Locality 2013, Number 2. http://journals.sfu.ca/nonlocality/inded.php/nonlocality/article/view/41/39.

[9] B. M. Vladimirskii, Biophysics 40 (1995) 915-923.

[10] A. A. Minakov, A. P. Nikolaenko, L. M. Rabinovich, Radiofizika 35 (1992) 488-497.

[11] B. T. Dotta, K. S. Saroka, M. A. Persinger, Neuroscience Letters 516 (2012) 54-56.

[12] K. S. Saroka, M. A. Persinger, Epilepsy and Behavior 28 (2013) 395-407.

[13] M. A. Persinger, K. S. Saroka, Journal of Electromagnetic Analysis and Applications 5 (2013) 151-155.

[14] L. J. Martin, S. A. Koren, M. A. Persinger, Pharmacology, Biochemistry and Behavior 78 (2004) 217-277.

[15] N. M. Murugan, M. A. Persinger, International Journal of Radiation Biology

[16] R. D. Pascual-Marqui, Methods In Experimental Clinical Pharmacology 24 (2002) 5-12.

[17] M. Cifra, J. Z. Fields, A. Farhadi, Progress in Biophysics and Molecular Biology 105 (2011) 233-246.

[18] T. Dahoun, S. Eliez, F. Chen, D. Badard, M. S. Schneider, F. Laroi, M. Debbane, Frontiers in Human Neuroscience 7 (2013) 329-339.

[19] R. A. Lanius, P. S. Williamson, R. L. Bluhn, M. Densmore, K. Boksman, R. W.J. Neufeld, J. S. Gati, R. S. Menon, Biological Psychiatry 57 (2005) 873-884.

[20] D. C.Parantie, J. Wu, J. M. Koller, A. Lim, S. L. Warren, K. Black, M. Sadler, N. H. White, T. Hershey, Diabetes Care 30 (2007) 2331-2337.

[21] S. Dehaene, N. Tzourio, V. Frak, L. Raynaud, L. Cohen, J. Mehler, B. Mazoyear, Neuropsychologica 34 (1996) 1097-1106.

[22] M. A. Scott, M. A. Persinger, Journal of Signal and Information Processing 4 (2013) 282-287.

[23] M. A. Persinger, International Letters of Chemistry, Physics and Astronomy 2 (2014) 1-10.

[24] M. N. Murugan, L.M. Karbowski, M. A. Persinger, Water 6 (2014) 45-60. 